\title{
Protective effects of sodium ferulate on flap transplantation via anti-inflammatory modulation and oxidative stress inhibition
}

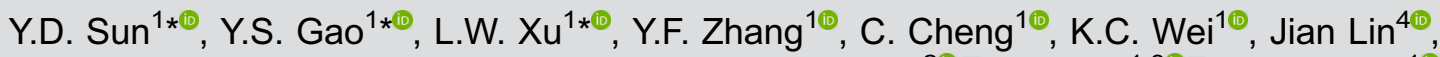 \\ G. Chen ${ }^{2 \oplus \bowtie}$, C.Y. Liu ${ }^{1,3 \oplus 凶}$, and Q.F. Li ${ }^{10}$ \\ ${ }^{1}$ Department of Plastic Surgery, Shanghai Ninth People's Hospital, Shanghai Jiao Tong University School of Medicine, \\ Shanghai, China \\ ${ }^{2}$ Department of Plastic Surgery, Jiangsu Province Hospital of Traditional Chinese Medicine, Nanjing, China \\ ${ }^{3}$ Department of Plastic Surgery, Changzheng Hospital of Navy Medical University, Shanghai, China \\ ${ }^{4}$ Department of Orthopedics, Xinhua Hospital (Chongming) Affiliated to Medical College, Shanghai Jiao Tong University, \\ Shanghai, China
}

\begin{abstract}
Ischemia-reperfusion injury (IRI) has brought attention to flap failure in reconstructive surgery. To improve the prognosis of skin transplantation, we performed experimental IRI by surgical obstruction of blood flow and used sodium ferulate (SF) to prevent IRI in rats. After SF treatment, the morphological and histological changes of the skin flaps were observed by H\&E and Masson's trichrome staining. We also detected the expression levels of COX-1, HO-1, and Ki67 by immunohistochemical and western blot analysis. Moreover, enzyme-linked immunosorbent assay was used to identify the content of tumor necrosis factor (TNF)- $\alpha$, myeloperoxidase (MPO), malondialdehyde (MDA), and nitric oxide (NO) in peripheral blood and skin tissue. Compared with the model group, SF treatment significantly improved the recovered flap area (\%) and promoted collagen synthesis. Cyclooxygenase-2 (COX-2) expression was significantly inhibited by heme oxygenase-1 (HO-1) induction after SF treatment. Furthermore, SF significantly inhibited the levels of TNF- $\alpha$ in peripheral blood, MPO and MDA in the skin tissue, and the increased synthesis of NO. Our results showed the protective effects of SF on IRI after flap transplantation and we believe that the protective effects of SF was closely related to the alleviation of the inflammatory response and the inhibition of the oxidative stress injury.
\end{abstract}

Key words: Sodium ferulate; Flap transplantation; Ischemia-reperfusion injury; Inflammation; Oxidative stress

\section{Introduction}

Flap transplantation is the main modality in wound repair and reconstructive surgery, and partial ischemia and necrosis of the flap is the most common complication in reconstructive surgery (1). Inadequate hemoperfusion and ischemia-reperfusion injury (IRI) after transplantation are considered to be the major cause of flap necrosis and failure $(2,3)$. Therefore, an effective way to improve the recovery of skin transplantation is important $(4,5)$. With the deepening of research on mechanisms of ischemia and hypoxia injury, IRI prevention and treatment are becoming the promising tools for us to explore.

Traditional Chinese Medicine (TCM) encompasses a wide range of practices and has gained excellent feedback from Asian people in their physiological restoration
(6). Among them, angelica has been shown to improve blood circulation and eliminate stasis (7). Studies have shown that angelica has good therapeutic effects on hypoxia and ischemia injury (8). Studies have proven that sodium ferulate (SF, the brand name is Angelicone) is one of the effective components of angelica extract, which is a type of new non-peptide endothelin receptor antagonist. SF has beneficial effects in microcirculation through activating the anti-inflammatory system, enhancing anticoagulation modulation, increasing the synthesis of nitric oxide (NO), and preventing lipid peroxidation injury (8-11).

SF has been widely used in the treatment of angina pectoris, cerebral infarction, and renal ischemic lesions. It was also proven to be effective in the treatment of diabetic

Correspondence: C.Y. Liu: <yueyue1888@hotmail.com > | G. Chen <njcg110@163.com>

${ }^{*}$ These authors contributed equally to this work.

Received October 13, 2020 | Accepted February 2, 2021 
foot and chronic skin ulcers $(12,13)$. However, the effects of SF on flap transplantation remain unknown. In this study, we investigated the effects of SF on the percentage of the recovered flap area after IRI by establishing an animal model of skin flap transplantation in rats. We explored the related mechanism of SF effects and provided an experimental basis for its subsequent clinical application.

\section{Material and Methods}

\section{Experimental animals}

Male Sprague Dawley rats (6-8 weeks, 230-270 g) were purchased from the Experimental Animal Center of General Hospital of Nanjing Military Region and were subjected to experimental IRI. The animals were fed with mixed feed, given natural light exposure in a single cage with the temperature being controlled at about $25^{\circ} \mathrm{C}$. The animals were adapted for one week before the experiment. At $8-10$ weeks of age, the rats were randomly divided into 3 groups as follows: sham surgery $(n=24)$, model group with experimental IRI $(n=24)$, and SF treatment group $(n=24)$. All animal procedures performed in this study were in accordance with the ethical standards of the Ethics Committee and ARRIVE guidelines. The approval number of Ethics committee is IACUC-2003125.

\section{Experimental reagents}

SF was purchased from Nanjing Simcere (China, National Medicine Permit No. H20065557). Myeloperoxidase (MPO), NO, and malondialdehyde (MDA) detection kit were purchased from Nanjing Jiancheng Biotech Corp (China). The antibody of cyclooxygenase (COX-2), heme oxygenase (HO-1), and Ki67 were purchased from the British Abcam company. The ELISA test kit for tumor necrosis factor (TNF)- $\alpha$ in rats was purchased from Shanghai JiaTai Biotech Corp. (China).

\section{Experimental IRI in rats}

Experimental IRI was induced by surgical intervention of the blood flow of the flap and the sham-operated rats were used as control. Briefly, we used $3 \%$ sodium pentobarbital for intraperitoneal anesthesia $(0.15 \mathrm{~mL} / 100$ g body weight). In the sham group (CTL group), the flap was sutured in situ and no other treatment was performed. In the model and SF treatment groups, we used inferior epigastric artery skin flap as a flap ischemia-reperfusion injury model, with the area of the axial flap about $3 \times 6 \mathrm{~cm}^{2}$. Thereafter, we blocked the blood flow with a microvascular clamp immediately after the establishment of the superficial epigastric flap for $8 \mathrm{~h}$. After that, surgical microscopy was performed to confirm the recovery of blood supply. In the SF group, SF was administered into the abdominal cavity at $80 \mathrm{mg} / \mathrm{kg}$ per day on the day before surgery, and for 7 days after surgery (the concentration of SF solution was $1 \%, 2.5 \mathrm{~mL}$ ). The rats in the model group were given the same amount of saline intraperitoneally according to the same procedure.

\section{Indicators}

In each group, 6 rats were randomly selected for observation at $8,24 \mathrm{~h}$, and 3 and 7 days after IRI surgery. The percentage of the recovered flap area was calculated by the recovered area compared to the total skin flap area, and the total surface area of the flaps was traced. We analyzed the areas using the ImageJ software $(\mathrm{NIH}$, USA). The flap tissue was equally divided along the central point of the flap (the diagonal intersection was the center), fixed with morphological method, and made into tissue homogenate to detect the levels of MPO, NO, and MDA by enzyme-linked immunosorbent assay (ELISA) and the nitro blue tetrazolium (NBT) method. At the same time, the peripheral blood of rats was collected, and ELISA was used to detect the level of TNF- $\alpha$ in serum.

\section{Histological and immunohistochemical assessments}

After the experiment, the rats were killed under isoflurane anesthesia and the flap tissue was washed in PBS. Skin tissue was fixed with $4 \%$ paraformaldehyde (PFA) and embedded in paraffin. Serial longitudinal sections were cut with a thickness of $5 \mu \mathrm{m}$, then deparaffinized, rehydrated, and antigen retrieved. Nonspecific immunoreactivity was blocked by incubation with normal BSA serum for $1 \mathrm{~h}$ at room temperature, and sections were incubated with anti-COX2, anti-HO-1, and anti-Ki67 antibodies (1:1000), incubated overnight at $4^{\circ} \mathrm{C}$, followed by treatment with the streptavidin-peroxidase kit according to the manufacturer's instructions (Sangon Biotech, China). The sections were also stained with hematoxylin and eosin (H\&E) to evaluate the morphological changes of the flap. As a control, a subset of sections was incubated with PBS instead of the primary antibody. The expression of COX2, HO-1, and Ki67, represented by the staining intensity under $\times 400$ magnification, was assessed by double-blind evaluation.

\section{Western blot analysis}

Skin samples were homogenized in RIPA lysis buffer with $1 \%$ phosphatase and protease inhibitor cocktail (Sigma-Aldrich, USA). The mixture homogenates were centrifuged $\left(12,000 \mathrm{~g}, 15 \mathrm{~min}, 4^{\circ} \mathrm{C}\right)$ and the supernatants were collected. Protein concentration was measured following Bradford's colorimetric method. Furthermore, the supernatants were mixed with a $5 \times$ SDS/PAGE sample buffer. Equal amounts of proteins $(20 \mu \mathrm{g})$ were separated by a $10 \%$ SDS/PAGE gel and then transferred to PVDF membranes (Merck Millipore, USA). After blocking in a $5 \%$ BSA buffer for $1.5 \mathrm{~h}$, membranes were incubated at $4{ }^{\circ} \mathrm{C}$ overnight with specific primary antibodies for COX2, HO-1, and GAPDH at a dilution of 1:000 (Cell Signaling Technology, USA). After washing with TBST three times, membranes were incubated with a 
secondary antibody (Cell Signaling Technology). For visualization of the bands, all membranes were incubated with Immobilon Western Chemiluminescent HRP substrate (Millipore) for desired durations.

\section{Statistical analysis}

All data were processed by SPPS (IMB, USA) 13.0 statistical software, and the data are reported as means \pm SD. Student's $t$-tests (unpaired, two-way) were applied to compare sham versus treatment groups. Statistical significance was set at $\mathrm{P}<0.05$.

\section{Results}

\section{SF treatment increased the recovered skin flap area}

In the image from the control group, both the epidermis and dermis appeared normal and intact. Additionally, no animals died and no blood accumulation, effusion, or infection occurred in the sham group (Figure 1A). However, compared with the sham group, necrosis and limited recovered zones were observed in the distal end of the flap 3 days post-surgery in the IRI group. Seven days post-surgery, there was partial ulceration and scabs formed on the distal end of the flaps (Figure 1A). In contrast, after SF treatment, the necrosis area of the skin in the SF group was significantly less than that in the model group. The recovered flap area (\%) also increased after SF treatment (Figure 1B). Therefore, SF treatment had a protective effect on preventing IRI, increasing the percentage of the recovered flap area (Figure 1).

\section{SF served as anti-inflammatory modulator}

In the model group, the inflammatory reaction of the skin graft was severe, with obvious inflammatory cell infiltration, and large necrotic areas compared with the sham group at different points of time after IRI (Figure 1C). However, at the same experimental time point, SF samples appeared histologically normal and the inflammatory reaction was attenuated. Masson's trichrome staining also revealed that SF prevented collagen loss and increased its synthesis after the IRI (Figure 1C). To confirm this finding, we tested two markers, COX-2 and $\mathrm{HO}-1$, which play important roles in inflammatory reaction and oxidative stress response (14). Through COX-2 immunohistochemical staining, we observed that the degree of inflammation in the model group significantly
A
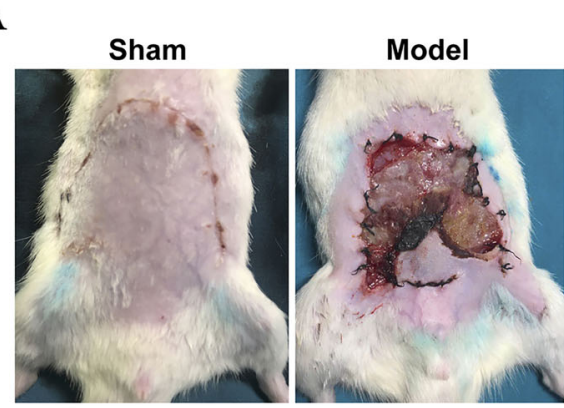

$\mathrm{C}$

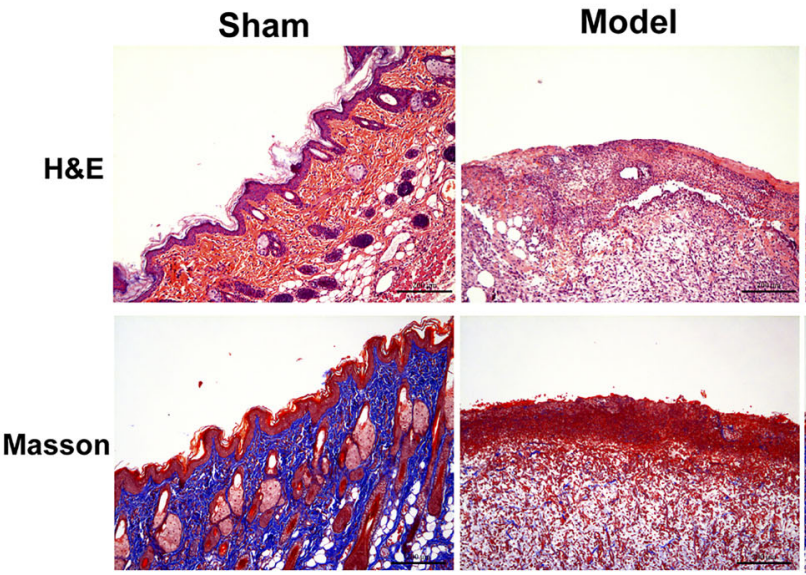

SF

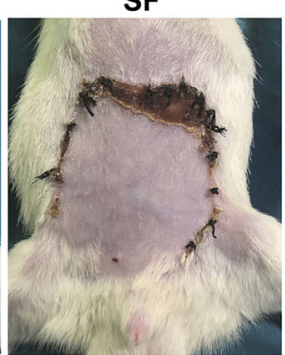

B $\rightarrow$ sham

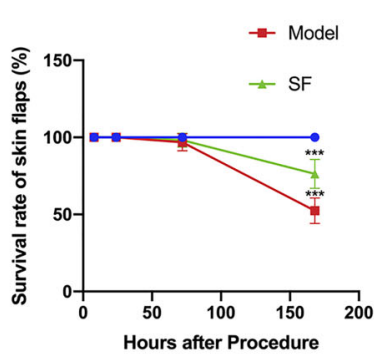

SF

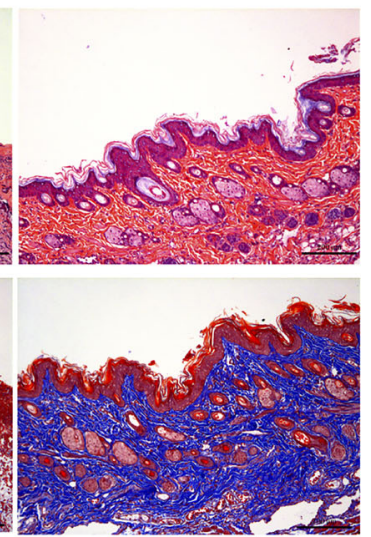

Figure 1. Morphological images of the A) lesion area and B) comparison of the percentage of the recovered skin flap area (\%, mean \pm $\mathrm{SD}, \mathrm{n}=6$ per group) after 7 days of establishment of the ischemia-reperfusion model in each group ( ${ }^{* * *} \mathrm{P}<0.001$ compared to Sham, Student's $t$-test). C, H\&E staining of the flap tissue (top). Masson staining (bottom) suggests the improved synthesis of the collagen after sodium ferulate (SF) treatment. Scale bar, $200 \mu \mathrm{m}$. 


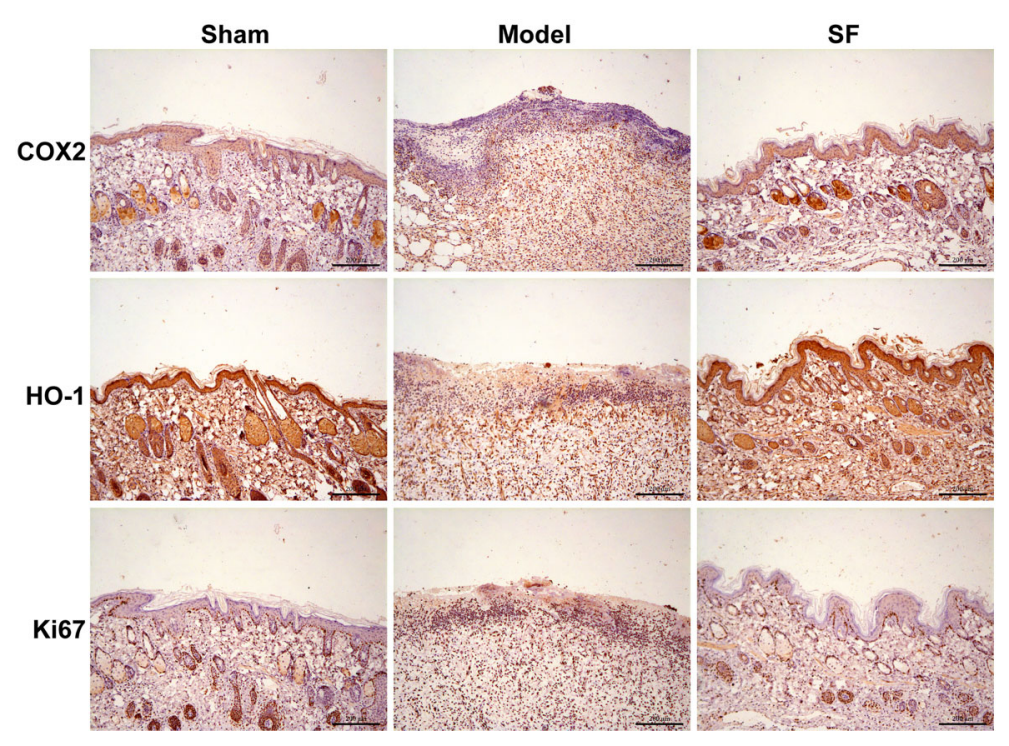

Figure 2. Assessment of the immunohistochemical staining of the flap tissue in each group: sham surgery, model group with experimental ischemia-reperfusion injury, and sodium ferulate (SF) treatment group. The SF group presented up-regulation of cyclooxygenase-2 (COX-2), heme oxygenase-1 (HO-1), and Ki67 markers compared with the model group. ( $\mathrm{n}=6)$. Scale bar, $200 \mu \mathrm{m}$.

increased after IRI surgery. In comparison, HO-1 decreased its expression due to severe inflammation (Figure 2). However, SF treatment resulted in a significantly decreased expression of COX-2 in the dermis, which means the inflammatory cytokines signal pathway was inhibited. Consistent with that, the expression level of antioxidant enzyme $\mathrm{HO}-1$ was significantly increased in the SF group at 7 days after surgery. Consistent results were found by western blot analysis and SF showed outstanding protective effects to reverse the damage by IRI (Figure 3). These results suggested COX-2 expression was inhibited after SF treatment by $\mathrm{HO}-1$ induction. Furthermore, SF had obvious regulatory functions on both inflammatory reaction and oxidative stress in the IRI model.

\section{IRI induced up-regulation of MPO, MDA, and NO and decreased TNF- $\alpha$}

We observed that the MPO and MDA concentrations increased after IRI surgery compared to the sham group (Figure 4A and B). Treatment with SF resulted in a significantly decreased expression of these two markers in tissue homogenate $(P<0.05)$ (Figure $4 A$ and $B$ ). In contrast, the NO concentration in tissues showed that the SF group was significantly higher than that of the model group and the sham group, while the difference between the model group and the sham group was not significant (Figure $4 \mathrm{C}$ ). Additionally, at $24 \mathrm{~h}$ after surgery, the TNF- $\alpha$ concentration in peripheral blood was reduced significantly to a relatively normal level in the SF group (Figure 4D). These results further suggested that, consistent with our previous results, SF could efficiently protect against

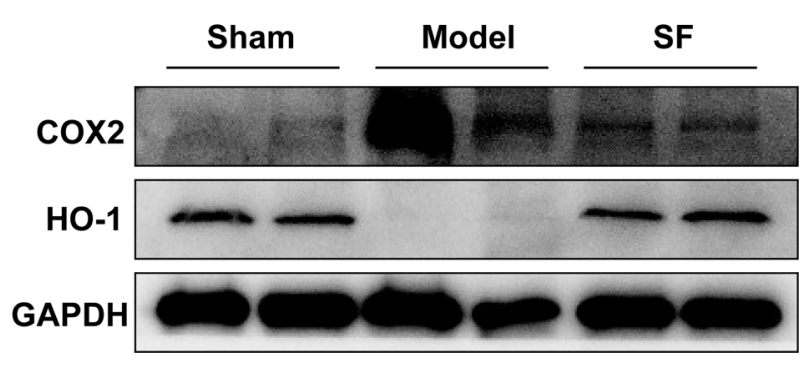

Figure 3. Western blot analysis results show the activation of cyclooxygenase-2 (COX2) expression and down-regulation of heme oxygenase-1 (HO-1) expression in model group (lanes 3 and 4) compared to Sham. After the sodium ferulate (SF) treatment, the results (lanes 5 and 6 ) were reversed and the COX2 and $\mathrm{HO}-1$ expression levels were similar to the sham group (lanes 1 and 2).

the inflammatory effects of IRI surgery shedding light on the positive effects of SF's application on IRI.

\section{Discussion}

Regarding the molecular mechanism of flap IRI, research has shown that oxidative free radicals, intracellular calcium overload, microvascular injury, infiltration of inflammatory cells, and the release of related inflammatory cytokines play important roles in the ischemiareperfusion process $(15,16)$. Oxygen free radicals can attack unsaturated fatty acids in biofilm and trigger lipid peroxidation to produce lipid peroxides, including aldehyde groups (MDA), keto, hydroxyl, carbon, and new free 

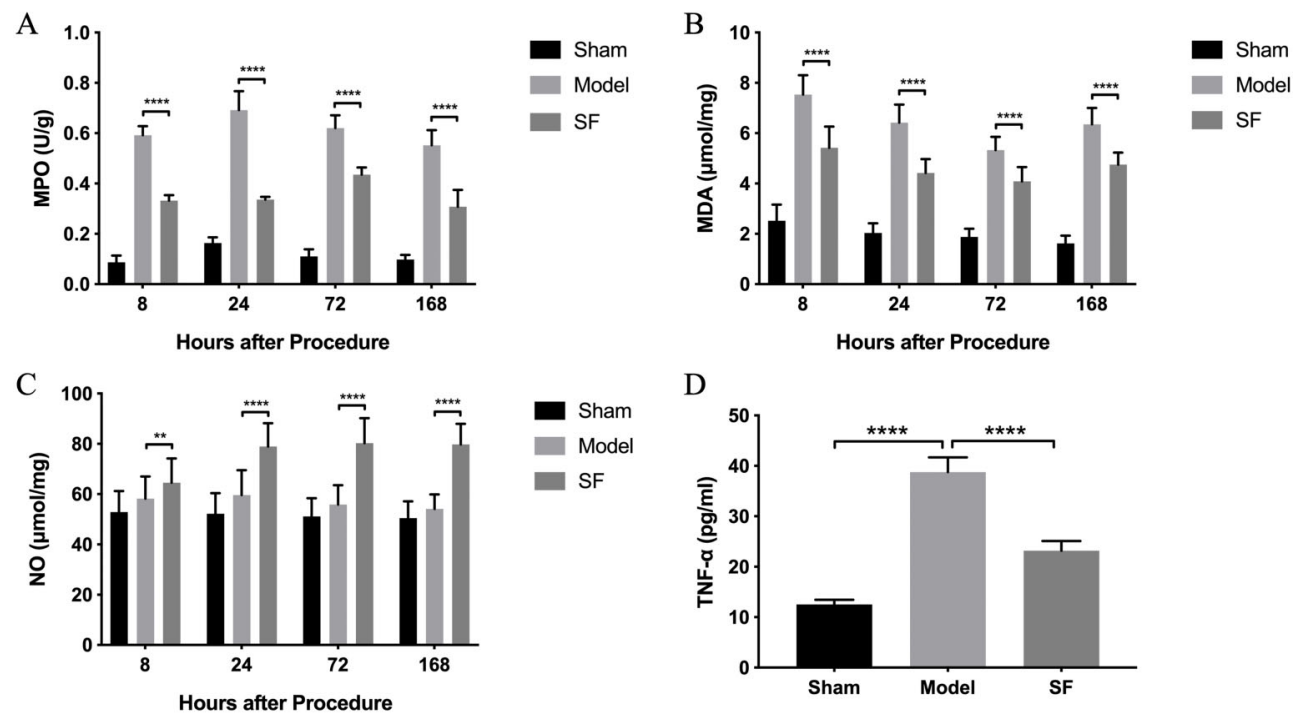

Figure 4. Myeloperoxidase (MPO), malondialdehyde (MDA), and nitric oxide (NO) changes in flap tissue. Tumor necrosis factor (TNF)- $\alpha$ levels in peripheral blood of sham surgery group, model group with experimental ischemia-reperfusion injury, and sodium ferulate (SF) treatment group (mean $\pm \mathrm{SD}, \mathrm{n}=6$ ). ${ }^{* \star} \mathrm{P}<0.05$, ${ }^{* \star * *} \mathrm{P}<0.001$, Student's $t$-test.

radicals, etc. When the release of free radicals exceeds the ability of the cell to repair itself, the cell can initiate the apoptotic pathway and ultimately cause tissue damage $(7-10,17)$.

SF is the sodium salt of ferulic acid (4-hydroxy-3methylcinnamic acid). The formula of ferulic acid is $\mathrm{C}_{10}$ $\mathrm{H}_{10} \mathrm{O}_{4}$ and its molecular weight is 194 (10). SF is a nonpeptide endothelin receptor antagonist, which can inhibit endothelin-induced vasoconstriction, blood pressure and vascular smooth muscle cell proliferation, reduce vascular endothelial damage, inhibit platelet aggregation, scavenge free radicals, and prevent lipid peroxidation. In the cardiovascular and cerebrovascular systems, digestive system, urinary system, central and peripheral nervous systems, visual system, and in ischemia-reperfusion injury in both animal experiments and clinical studies, SF shows large potential and versatile protective roles $(18,19)$.

This study confirmed that SF can significantly improve the recovered flap area (\%) after IRI. Its mechanism may be due to the following aspects. First, SF has a role in the regulation of oxidative stress, which can improve the balance between $\mathrm{NO}$ and endothelin after IRI, and improve microcirculation (20). In normal tissues, endothelin and NO are in a state of balance. NO can counteract the vasoconstriction of endothelin, inhibit the formation of microthrombus, and avoid the occurrence of disseminated intravascular coagulation (8,17-19). We found that the NO levels increased significantly in the SF group, and the mechanism of this phenomenon may be due to the specific endothelin receptor antagonist effect. Secondly, SF can reduce the formation of tissue MPO and MDA.
MPO is the peroxidation product formed after polyunsaturated fatty acid metabolism, which is oxidized by the active oxygen in vivo. Therefore, the concentration of this marker can reflect the amount of free radicals produced in vivo. Thus, it also indicates the degree of damage of oxidative stress to the tissue. The results of this experiment showed that SF alleviated damage by oxygen free radicals and reduced lipid peroxidation injury caused by ischemia-reperfusion. In addition, SF reduced the inflammatory response caused by leukocyte infiltration. Leukocyte infiltration can mediate microvascular injury, which contributes to the pathogenesis of IRI. Neutrophils are activated, which adhere to vascular endothelial cells and cause the mechanical blockage of microvessels. They are also important sources of MPO, so an excess of neutrophils can increase MPO content in local tissues (8,17-19).

This study found that during the process of IRI, the infiltration of inflammatory cells, such as neutrophils, in the flap tissue of the SF group was significantly decreased and the content of MPO was significantly decreased. In addition, the level of TNF- $\alpha$ in peripheral blood of the SF group was also significantly lower than the sham group, indicating that SF had a significant anti-inflammatory effect and inhibited neutrophil chemotaxis and aggregation in inflammation. Furthermore, SF may also inhibit cell apoptosis by reducing calcium overload. The protective effect of SF on a variety of cells has been reported. Liu et al. (21) has shown that SF relieves myocardial injury caused by $\mathrm{Ca}^{2+}$ overload, reduce myocardial calcium overload caused by IRI, and improve cardiac function. 
Li et al. (22) found that SF reduces the expression level of MDA and BAX protein in muscle tissue of the experimental group and reduces apoptosis after IRI. Although there is no direct evidence about the effect of SF on skin cells, in this experiment, we also observed that SF promoted the injured skin flap to recover histologically, indicating that SF may have a protective effect on normal skin cells.

\section{References}

1. Lin J, Zhou F, Sun YD, Gao YS, Li HZ, Zheng HP, et al. Modified anterior tibial artery perforator-pedicled propeller flap for soft-tissue coverage of the ankle and heel. World J Surg, 2020; 44: 2237-2242, doi: 10.1007/s00268-02005452-y.

2. Cetin C, Kose AA, Aral E, Colak O, Ercel C, Karabagli $Y$, et al. Protective effect of Fucoidin (a neutrophil rolling inhibitor) on ischemia reperfusion injury: experimental study in rat epigastric island flaps. Ann Plast Surg 2001; 47: 540546, doi: 10.1097/00000637-200111000-00012.

3. Wang $\mathrm{H}$, Li Z, Liu X. Effects of different durations and times of ischemic preconditioning on ischemia-reperfusion injury to tram flaps in rats [in Chinese]. Zhongguo Xiu Fu Chong Jian Wai Ke Za Zhi 2006; 20: 431-433.

4. Badhwar A, Bihari A, Dungey AA, Scott JR, Albion CD, Forbes $T L$, et al. Protective mechanisms during ischemic tolerance in skeletal muscle. Free Radic Biol Med 2004; 36 : 371-379, doi: 10.1016/j.freeradbiomed.2003.11.015.

5. Rah DK, Min HJ, Kim YW, Cheon YW. Effect of platelet-rich plasma on ischemia-reperfusion injury in a skin flap mouse model. Int J Med Sci 2017; 14: 829-839, doi: 10.7150/ ijms.19573.

6. Cheung F. TCM: Made in China. Nature 2011; 480: S82S83, doi: $10.1038 / 480 S 82 a$.

7. van den Heuvel MGW, Buurman WA, Bast A, van der Hulst RRWJ. Review: ischaemia-reperfusion injury in flap surgery. J Plast Reconstr Aesthet Surg 2009; 62: 721-726, doi: 10.1016/j.bjps.2009.01.060.

8. Li M, Wu LY, Zhu LL, Fan M. Effective components from angelia on hypoxia injury: research progress [in Chinese]. Bull Acad Milit Med Sci 2008; 32: 87-90.

9. Chen JT, Yang CR. Researches in Angelica $L$ [in Chinese]. Nat Product Res Develop 2004; 16: 359-365.

10. Wang $\mathrm{BH}, \mathrm{Ou}-\mathrm{Yang} \mathrm{JP}$. Pharmacological actions of sodium ferulate in cardiovascular system. Cardiovasc Drug Rev 2010; 23: 161-172, doi: 10.1111/j.1527-3466.2005.tb001 63.x.

11. Chen HP, Liao ZP, Huang QR, He M. Sodium ferulate attenuates anoxia/reoxygenation-induced calcium overload in neonatal rat cardiomyocytes by NO/cGMP/PKG pathway. Eur J Pharmacol 2009; 603: 86-92, doi: 10.1016/j.ejphar. 2008.12.003.

12. Zhang Q, Zhao YH, Xu YH, Chen ZW, Liu NW, Ke C, et al, Sodium ferulate and $n$-butylidenephthalate combined with bone marrow stromal cells (BMSCs) improve the therapeutic effects of angiogenesis and neurogenesis after rat focal

\section{Acknowledgments}

This work was supported by the Science and Technology Committee of Shanghai Municipal Government (grant No.16YF1403500), the National Natural Science Foundation of China (grant No. 81701205 and 82072191), and the China Postdoctoral Science Foundation (grant No. 2018M630449).

cerebral ischemia. J Trans/ Med 2016; 14: 223, doi: 10.1186/ s12967-016-0979-5.

13. Cheng CY, Kao ST, Lee YC. Ferulic acid ameliorates cerebral infarction by activating Akt/mTOR/4E-BP1/Bcl-2 anti-apoptotic signaling in the penumbral cortex following permanent cerebral ischemia in rats. Mol Med Rep 2019; 19: 792-804, doi: 10.3892/mmr.2018.9737.

14. Li H, Zhou X, Zhang J. Induction of heme oxygenase-1 attenuates lipopolysaccharide-induced inflammasome activation in human gingival epithelial cells. Int J Mol Med 2014; 34: 1039-1044, doi: 10.3892/ijmm.2014.1865.

15. Werker PM, Kon M, Green CJ, Ambrose I, Hildago A. Oxidative processes and free radical scavengers in ischaemia-reperfusion injury in adipocutaneous flaps: in vitro lipid peroxidation assessment. Br J Plast Surg 1995; 48: 590596, doi: 10.1016/0007-1226(95)90050-0.

16. Moghimian M, Soltani M, Abtahi H, Shokoohi M. Effect of vtamin $\mathrm{C}$ on tissue damage and oxidative stress following tunica vaginalis flap coverage after testicular torsion. J Pediatr Surg 2017; 52: 1651-1655, doi: 10.1016/j. jpedsurg.2017.07.001.

17. Matés JM, Sánchez-Jiménez FM. Role of reactive oxygen species in apoptosis: implications for cancer therapy. Int $J$ Biochem Cell Biol 2000; 32: 157-170, doi: 10.1016/S13572725(99)00088-6.

18. Wu Z J, Yu J, Fang Q J, Lian J B, Lin M J, et al. Sodium ferulate protects against daunorubicin-induced cardiotoxicity by inhibition of mitochondrial apoptosis in juvenile rats. J Cardiovas Pharmacol 2014; 63: 360-368, doi: 10.1097/ FJC. 0000000000000056

19. Chen YB, Dai F, Lu Q. Advances in research on the protective mechanisms of sodium ferulate against flap's ischemia-reperfusion injury [in Chinese]. Chin J Aesthetic Med 2012; 21: 682-685.

20. Gao-Yan HE, Xie M, Gao Y, Huang JG. Sodium ferulate attenuates oxidative stress induced inflammation via suppressing NALP3 and NF-KB signal pathway [in Chinese]. J Sichuan University (Medical Science Edition) 2015; 46: 367.

21. Liu JC, Wan L, Shao LJ, He M. Protective effects and mechanisms of pharmacological preconditioning induced by sodium ferulate on isolated rat heart [in Chinese]. Chin Pharmacol Bull 2007; 23: 618-622.

22. Li B, Li JN, Wen Y. Protection of sodium ferulate for apoptosis of ischemia-reperfusion injury and expression of $\mathrm{Bcl}-2$, bax protein in rat hind limb [in Chinese]. China $\mathrm{J}$ Orthop Trauma 2005; 18: 97-99. 